\title{
Load-cell based smart floor panel for indoor localization applications
}

\author{
Chung-Hao Cheng ${ }^{\mathrm{a}^{*}}$, Ming-Feng $\mathrm{Wu}^{\mathrm{a}}$, Chin-Tien Huang ${ }^{\mathrm{b}}$, and Kuo-Shen Chen ${ }^{\mathrm{b}}$ \\ ${ }^{\mathrm{a}}$ Graduate Student $\quad{ }^{\mathrm{b}}$ Professor \\ Department of Mechanical Engineering, National Cheng Kung University, \\ No.1, Dasyue Rd., East Dist., Tainan City 701, Taiwan \\ *Corresponding Author: chunghao97@gmail.com
}

\begin{abstract}
Accurate position detection of indoor objects is crucial for many intelligent life applications. Indoor position detection can be commonly performed using some forms of touch-based floor system. The present study develops a force-sensing floor panel for the indoor localization of human beings and moving objects such as mobile robots. The detailed geometric parameters of the floor panel are determined by simulations. The motion signals are detected by four load cells located at the panel corners and are used to determine the position of the applied force by means of rigid body statics. In addition, a novel recursive algorithm, aiming for calibrating the floor due to possible assembly errors, is proposed to improve the localization accuracy of the panel after installation. Essential tests are performed to examine the system performance. The results indicate that the localization accuracy is approximately $1 \mathrm{~cm}$ after calibration and can detect people motion provided the walking frequency less than $2 \mathrm{~Hz}$. Finally, a demonstration also presented to highlight other possible applications such as center of gravity measurement of irregular objects.
\end{abstract}

Keywords: localization, load-cell, smart floor, touch panel, force-sensing.

\section{Introduction}

Accurate position detection of indoor objects is crucial for many intelligent life applications. For instance, accurate position information is vital for assorted tasks as navigation, feedback control, coordinated motion, collision avoidance, and trajectory planning. In addition, the accurate localization of individuals in indoor living spaces is desirable for such applications as indoor security or the care of the elderly or infirmed. Accordingly, the problem of realizing indoor localization schemes has attracted considerable attention in the past few years. However, due to positioning resolution problems, poor signal reliability, and intricate indoor environment designs, a flawless positioning solution has yet to be proposed.

Various schemes for indoor localization have been proposed, including GPS localization ${ }^{(1)}$, inertial navigation systems (INS) $)^{(2)}$, ultrasonic localization $^{(3)}$, vision-based systems $^{(4)}$, RFID-based systems ${ }^{(5)}$, and so on. However, GPS signals cannot penetrate buildings. Moreover, the accuracy of GPS-based systems is insufficient to meet the positioning requirements of many smart building and indoor robots. Ultrasonic localization schemes have a simple structure and the potential to achieve a high degree of positioning accuracy. However, their performance is adversely affected by reflection and Non-Line-of-Sight (NLOS) propagation. Similarly, INS suffers from signal drafting and cannot be used without an external position updating scheme.

Schmidt et al. ${ }^{(6)}$ developed a table containing embedded force sensors, in which the position of an object on the table surface was determined by processing the force sensor signals utilizing an algorithm based on the principles of rigid body mechanics. The results showed that the proposed system enabled not only the weight of the placed object but also its position and interaction events to be identified. In other words, the proposed method had the potential to overcome many of the problems associated with the traditional localization schemes described above. Nevertheless, the proposed table had the ability only to detect and track single static loading, which was insufficient to the practical applications in typical indoor localization systems. The localization algorithm based on the original rigid body statics should be modified for extending the applications. In the present study, a similar approach is 
taken in developing a floor panel for localizing the position of indoor mobile objects and individuals. Meanwhile, the localization algorithm is examined and revised to cover the possibility of multiple and moving objects. Finally, a prototype is constructed for evaluating the design performance, validating the localization algorithms, and exploring other feasible applications. Notably, the floor panel can also serve as a tool to measure the weight and center of gravity of solid objects with an irregular shape.

Fig. 1 shows the schematic concept of the smart floor localization system. An indoor space consists of numerous floor panels with four load cells mounted. Once an individual or an object moves on a floor panel, its coarse location can be determined by identifying the responding panel. This coarse determination can estimate the location accurate to about $1 \mathrm{~m}$. Next, the fine location can be further determined by examining the relative location on the particular panel using the proposed scheme presented in Section 2. This should be able to provide positioning accuracy down to $1 \mathrm{~cm}$ level. By this coarse-fine approach, localization of objects in a large working space with high accuracy is possible. Practically, the coarse determination is a routing work and this work should focus on the development of the fine localization step.

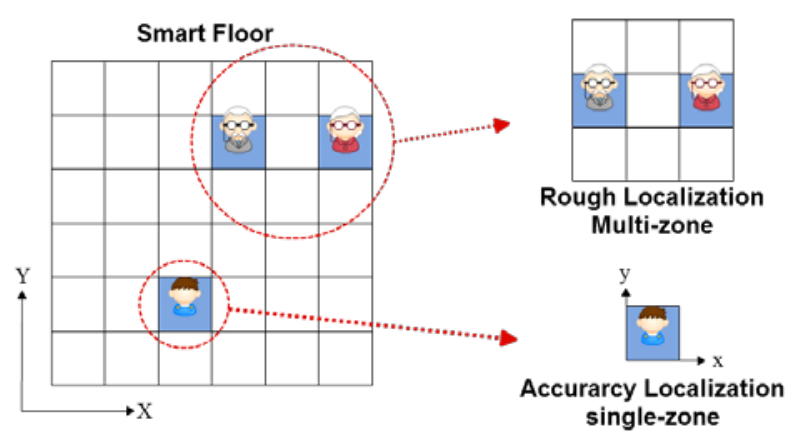

Fig. 1. The concept of smart floor for indoor localization.

The remainder of this paper presents the design, realization, and experimental validation of the panel in detail and is organized as follows. Section 2 describes both the localization methodology and the associated localization scheme, followed by the content in Section 3 of the design and implementation of the proposed smart floor panel, as well as proposing a recursive numerical algorithm for further improving the accuracy. Section 4 describes the experimental results and provides insights on the performance of the system. Section 5 demonstrates the practical applicability of the proposed system by working with an omnidirectional $\operatorname{robot}^{(7-8)}$ for robotic trajectory monitoring and uses this panel to serve as a tool to measure the center of gravity of solids with an irregular shape. Section 6 discusses the contributions and potential applications of the proposed floor panel. Finally, Section 7 provides some brief concluding remarks and indicates the intended direction of future research.

\section{Localization Methodology}

This section describes the use of the proposed force sensing technique to identify the position of single or multiple objects on the floor panel. To detect the position of an object on the floor panel surface, the center of pressure acting on the panel is determined based on the output signals of the four load cells.

Fig. 2(a) presents a schematic illustration of multi-object located on the floor panel. Consider the case of only a single object located on the panel surface at $\left(X_{1}, Y_{1}\right)$. Let the weight of this object be denoted as $W_{1}$. The setup assumes static forces, and hence, as shown in Eq. 1 and Fig. 2(a), $W$ is taken simply as the sum of the forces acting on the four load cells, i.e., $F_{1}, F_{2}, F_{3}$ and $F_{4}$. Note that here we assume each load cell behaves linearly and the transduction gains of all load cells are assumed to be identical.

Based on rigid body mechanics and moment equilibrium, the coordinates of the object, $\left(X_{1}, Y_{1}\right)$, can be determined using Eqs. (2) and (3). In turn, when an additional object is placed on the panel (for which the coordinates of the former object have already been determined), the same equations can be re-used to determine the coordinates of this new object. In other words, the panel has the ability to detect the position of both single objects and multiple objects via a simple algorithmic approach.

$$
W_{i}=\sum\left(F_{1 i}+F_{2 i}+F_{3 i}+F_{4 i}\right)
$$

$$
\begin{aligned}
& X_{i}=\left(\left(F_{2(i-1)}-F_{2 i}\right)+\left(F_{3(i-1)}-F_{3 i}\right)\right) \frac{x_{\max }}{\left(W_{(i-1)}-W_{i}\right)} \\
& Y_{i}=\left(\left(F_{3(i-1)}-F_{3 i}\right)+\left(F_{4(i-1)}-F_{4 i}\right)\right) \frac{y_{\max }}{\left(W_{(i-1)}-W_{i}\right)}
\end{aligned}
$$




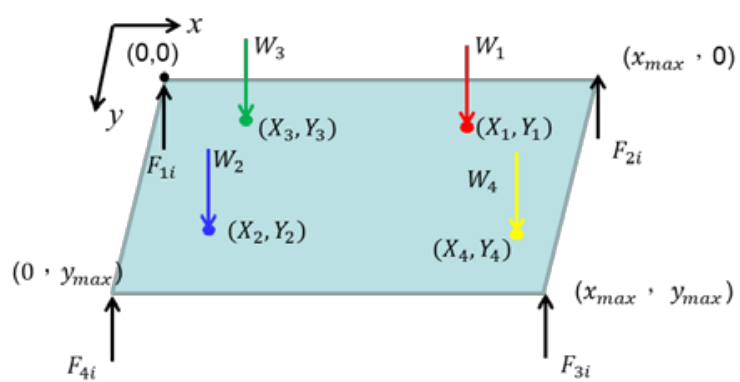

(a) Multi-load scheme.

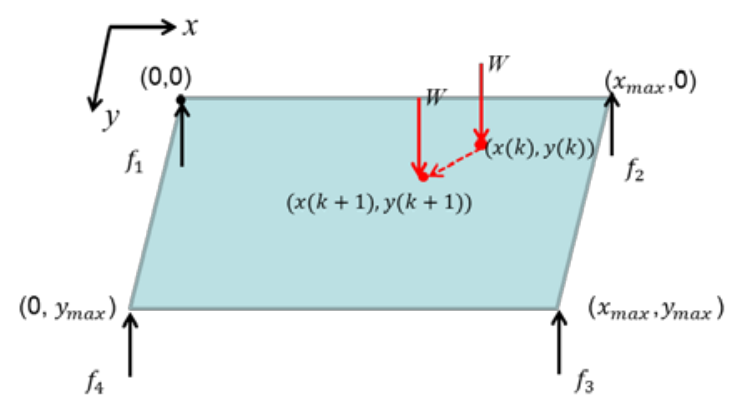

(b) Moving-load scheme.

Fig. 2. Schematic representation of proposed forcebased localization scheme.

Furthermore, the proposed scheme is also applicable for determining the position of a moving object on the panel under quasi-static movement. Fig. 2(b) shows that the new coordinates of this moving object can also be determined by the same equations (i.e., Eqs. (2) and (3)) for the time step, $k+1$, provided that the position at step $k$ is obtained. In addition, the applicability of this method subjected to dynamical motion is also considered and examined experimentally. The experimental details are elaborated in the subsequent section 4.4 .

\section{Smart Floor Panel Design}

This section commences by describing the details of design and overall system integration, including hardware and software, and proposing the associated system calibration, i.e., recursive numerical algorithm.

\subsection{Design}

As described earlier, load cells are the major sensing elements for realizing the localization. In this work, four Stype load cells are employed. Fig. 3 shows the picture of that load cell (S-100, JIHSENSE). The load cell has a measurement capability of $100 \mathrm{Kg}$ with a minimum resolution of $0.005 \mathrm{Kg}$. Each load cell is connected to a $6^{\text {th }}$ order low pass filter with a cutoff frequency of $60 \mathrm{~Hz}$ for attenuating undesirable high frequency signals and noise. The filtered signals are then amplified before being collected by an NI USB data acquisition system (NI-9215, 4-Channel Module, signal range $= \pm 10 \mathrm{~V}, 16$-Bit, Sampling rate $=100$ $\mathrm{kS} / \mathrm{s} / \mathrm{ch}$ ). Finally, the mechanics addressed in Section 2 is used to determine the force-applied location and a usermachine interface is also designed to visualize the final result. The entire flow is outlined in Fig. 4.

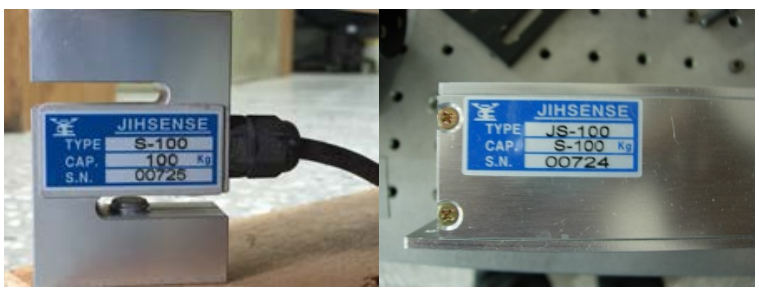

Fig. 3. JIHSENSE S load cell and amplifier.

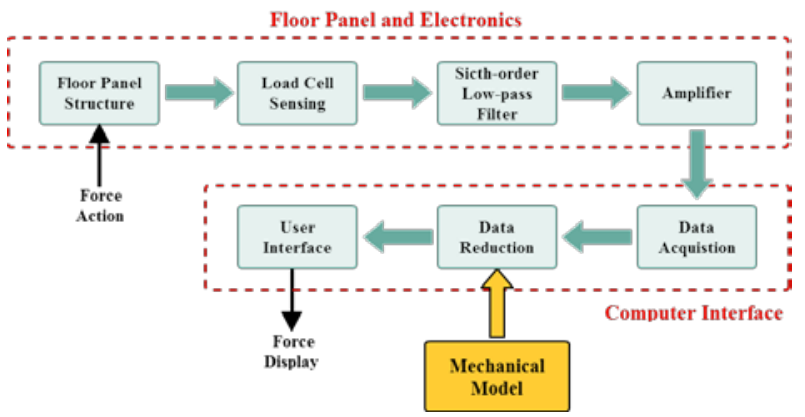

Fig. 4. The flowchart of the experimental system.

\subsection{Overall System integration}

Fig. 5 presents a schematic illustration of the overall system. In constructing the floor panel, the four load cells are sandwiched between an upper and lower plate and screwed in place such that they are located directly beneath the four corners of the panel. The placement of an individual or an object on the panel surface results in a change in the output signals of the four load sensors, from which the object weight can then be determined.

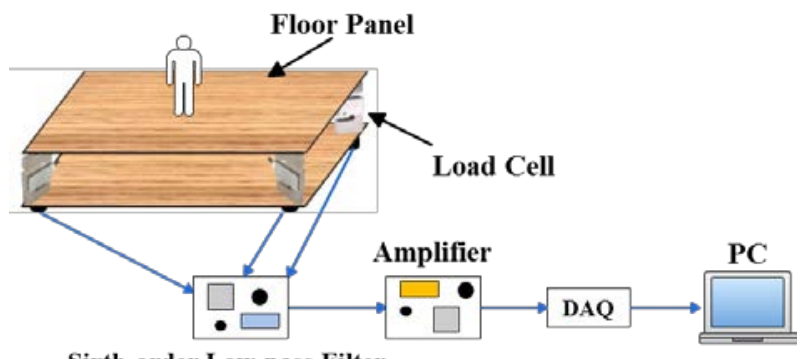

Sixth-order Low-pass Filter

Fig. 5. Schematic illustration of the overall system flowchart. 
A 3D finite element analysis using commercial available package Abaqus software is performed to optimize the dimension such as the thickness of the floor panel and to investigate the influence due to deformation during the service. Based on the analysis results and practical considerations such as available space, a square panel with a $1 \mathrm{~m}$ side length and $1 \mathrm{~cm}$ thickness made of plywood is chosen. Fig.6 shows a schematic and pictures of the force-sensing panel.

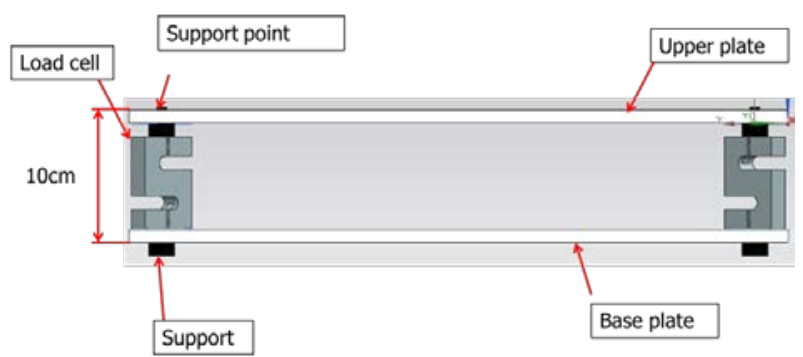

(a) Schematic illustration showing side-view of force-sensing floor panel.

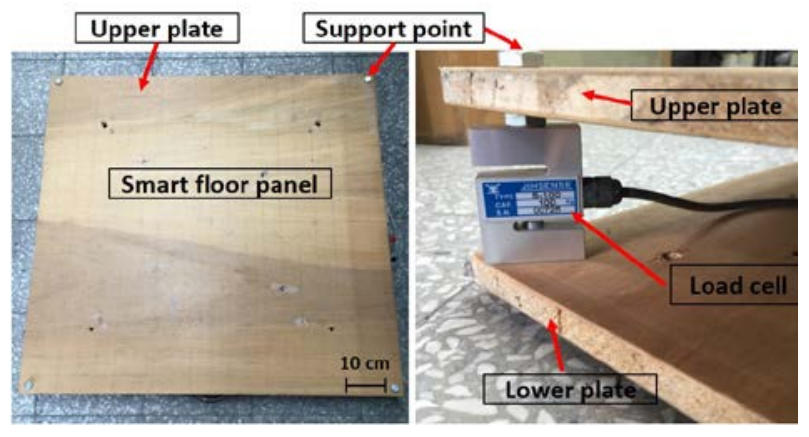

(b) Top view of panel and load cell position at corner of panel.

Fig. 6. Smart floor panel structure.

\subsection{System calibration}

In section 2, the sensor gains are assumed to be the same of all load cells force for performing the localization tasks. In practice, this is a dangerous assumption and the sensor gain should be individually calibrated. However, even this task is performed; other manufacturing, assembly, and handling errors could also provide additional sources of error to further degrade the localization accuracy. Consequently, an integrated calibration should be performed to improve the localization accuracy in a whole-system manner. Here a recursive numerical algorithm similar to our previous work in strain sensing ${ }^{(9-10)}$ is proposed to enhance the spatial resolution of the floor panel. Fig. 7 shows the essential flowchart of the calibration system. Two databases, which represent the original localization errors in $\mathrm{x}$ and $\mathrm{y}$ directions and must be obtained by off-line calibration, are required for initiating the process. The algorithm then uses the x-error map to gradually reduce the localization error in y-direction, and vice versa. Using a recursive manner, both errors are converged to two steady values.

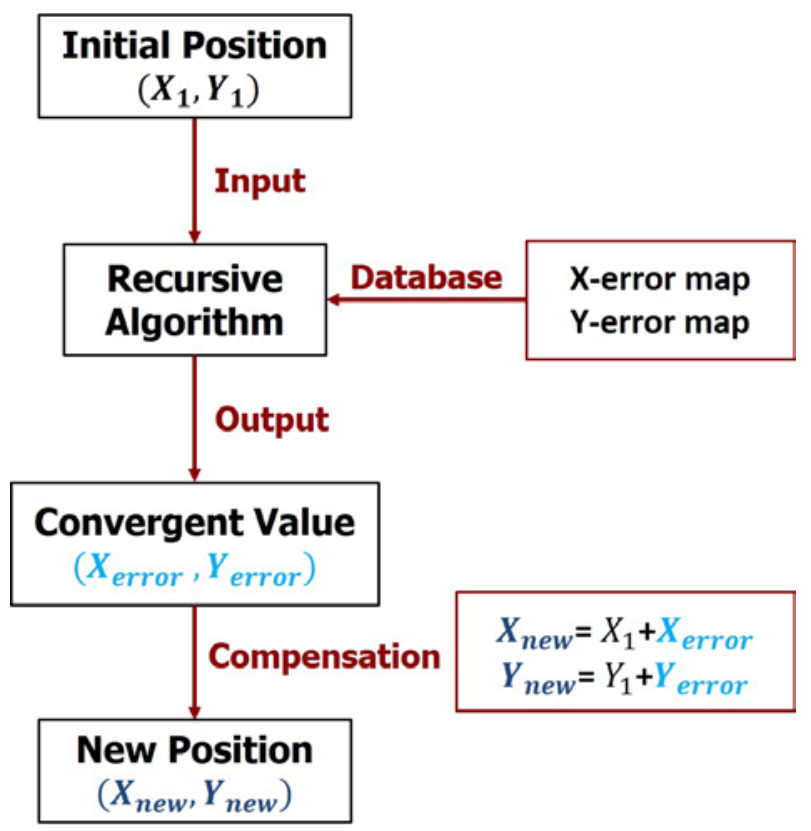

Fig. 7. The flowchart of the calibration system.

\section{Experimental Setup and Results}

This section addresses the experimental setup and presents the signal object localization result firstly, followed by multiple objects localization. Moreover, the capability of the system for tracking the position of a moving object is demonstrated. Finally, dynamic effects induced by the moving of individuals or robots on the panel is also considered and demonstrated.

\subsection{Single-load Static Scheme}

In the first experiment, a single object (a $3 \mathrm{~kg}$ free weight) was placed on the panel at various locations with a constant $\mathrm{y}$-coordinate position $(\mathrm{y}=50 \mathrm{~cm})$ and various $\mathrm{x}$ coordinate positions in the range of $x=10 \sim 90 \mathrm{~cm}$. Fig. 8(a) shows the original measurement results and the calibrated measurement results, respectively. It is seen that the original measurements have a poor accuracy, particularly at the extremities of the panel. Fig. 8(b) shows the localization errors of the original and calibrated measurement results. The results show that maximum localization errors can be reduced from $7.5 \mathrm{~cm}$ to $3 \mathrm{~cm}$ by using the calibration scheme and the localization performance is therefore significantly improved. 


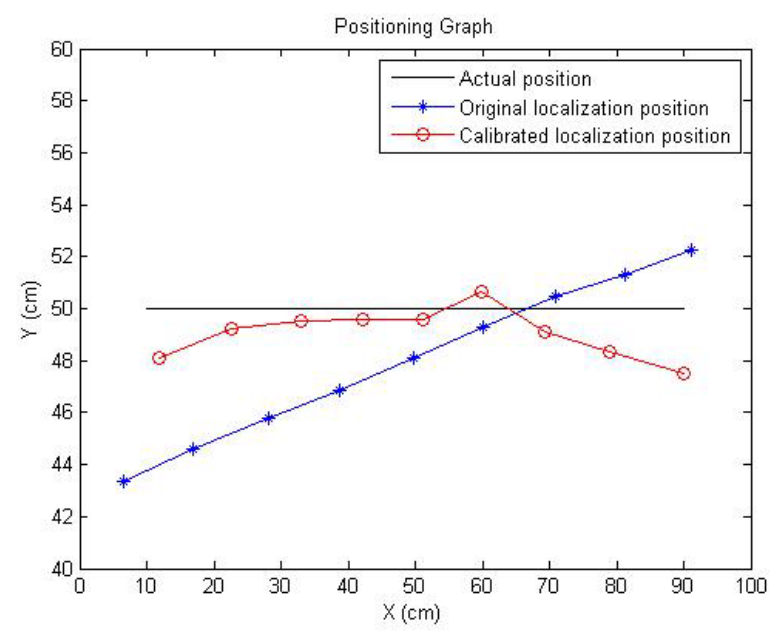

(a) Actual position, original localization position, and calibrated localization position in single-object placement tests.

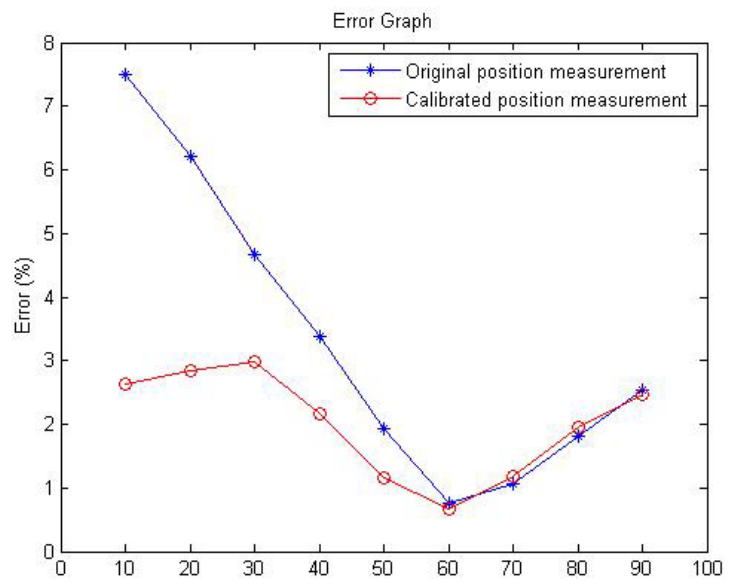

(b) Localization error of original position measurement and calibrated position measurement.

Fig. 8. The localization experimental results of singleload static scheme.

\subsection{Multi-load Static Scheme}

In the second experiment, three objects (A, B and C) were randomly placed in sequence on the floor panel, with each new object being added only once the location(s) of the previous object had been determined. The object positions and experimental localization results are shown in Fig. 9(a) and 9(b), respectively. As shown in Fig. 9(b), the localization error of the proposed scheme is equal to around $2 \mathrm{~cm}$ in both the $\mathrm{x}$ - and the $\mathrm{y}$-axis directions.

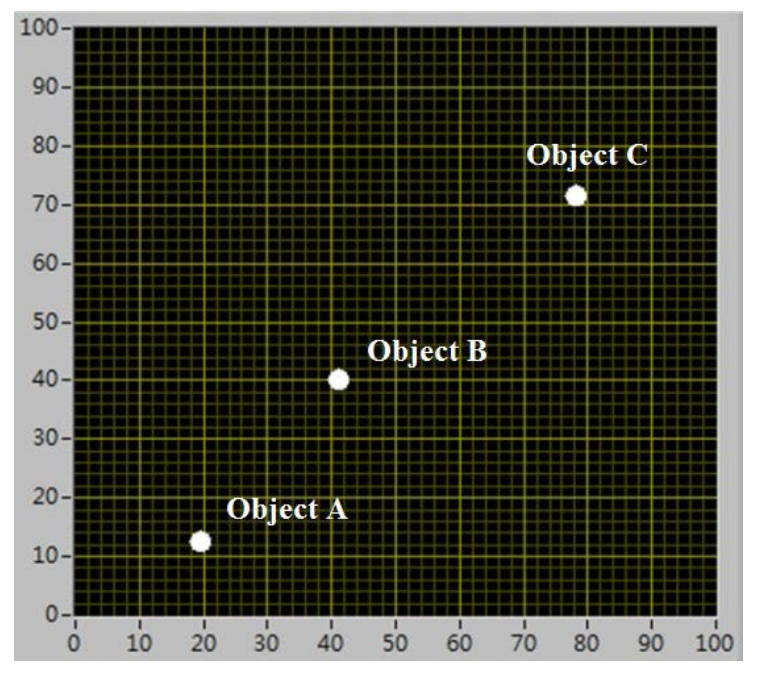

(a) Locations of objects A, B and C placed sequentially on panel surface.

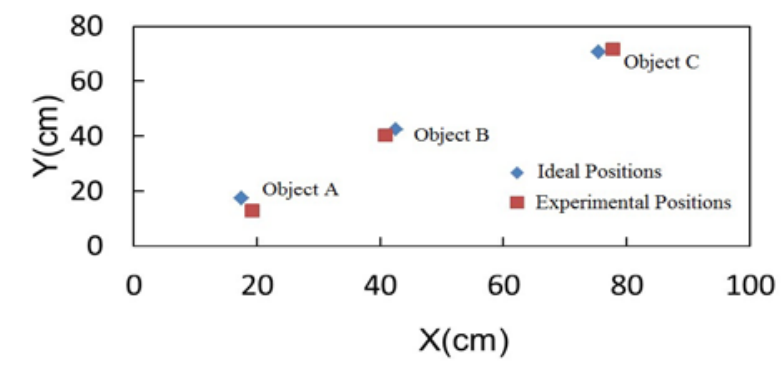

(b) Comparison of actual object position and experimental localization position.

Fig. 9. The localization experimental results of multiload static scheme.

\subsection{Moving-load Scheme}

In real smart panel applications, the aim is generally to detect the moving position of an individual continuously within a 2-D living or working space. In this study, the suitability of the proposed scheme for such an application was investigated by manually moving an object (a $3 \mathrm{~kg}$ free weight) along an arbitrary trajectory.

Fig. 10(a) shows the trajectory along which the object was moved during the tracking test. Fig. 10(b) presents the corresponding localization results. It is seen that the calculated trajectory deviates from the actual trajectory by no more than approximately $3 \mathrm{~cm}$. Notably, this localization error comprises a small manual positioning error component, and is thus reduced if the object is more accurately placed on the panel. Overall, the results show that the localization scheme has the ability to track the path of a moving object successively with a resolution acceptable for most intelligent life applications. 


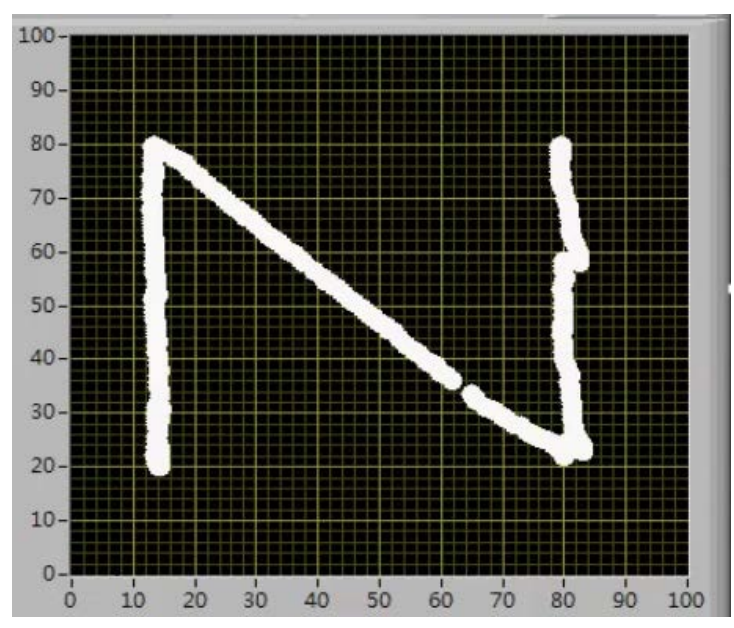

(a) Arbitrary trajectory of moving object

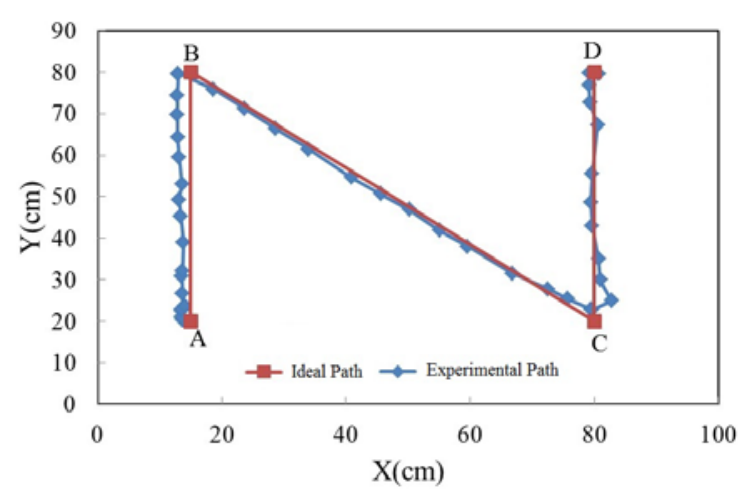

(b) Deviation of measured path from ideal path.

Fig. 10. The localization experimental results of moving-load scheme.

\subsection{Dynamic Scheme}

The fundamental mechanism for the proposed localization is based on statics and should not be able to count any dynamic effect. However, if the force-applying frequency, such as the walking rate, is not high and the excited dynamics can be quickly attenuated to a quasi-static state, the principle is still applicable.

A dynamic loading is applied by placing a box $(13 \mathrm{Kg})$ plunked down on the floor panel from a height of $1 \mathrm{~cm}$ to simulate the momentary force of human walking. The results, as shown in Fig. 11, indicate that initially the localization algorithm cannot be applicable due to dynamic effect. Nonetheless, after approximately $0.5 \mathrm{~s}$, the result is gradually converged to its true value as the dynamic effect decays. Practically, if the damping can be increased by mechanical re-design, the time required for converging is expected to be further reduced and therefore enhance the operating bandwidth. This could be the immediate work to be taken.

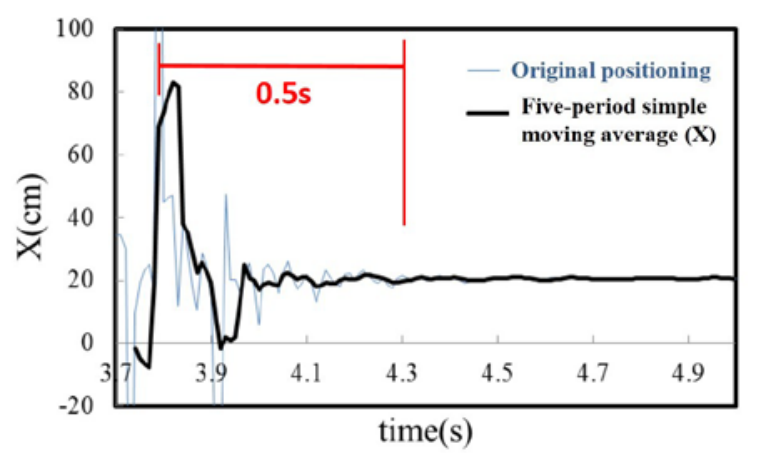

(a) X-axis position measurement

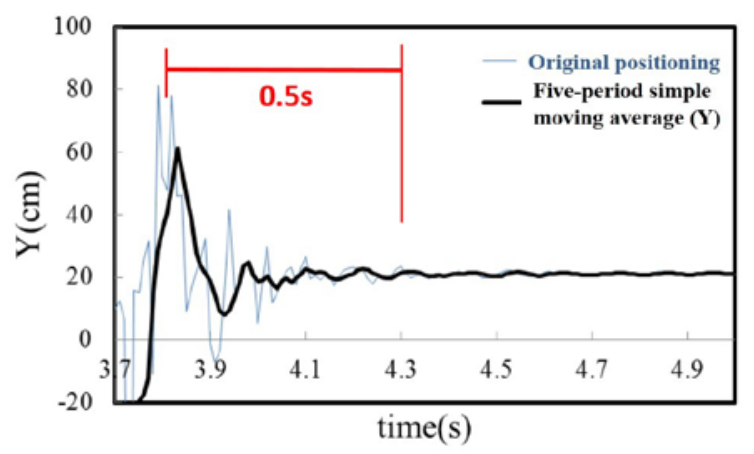

(b) Y-axis position measurement

Fig. 11. Dynamic scheme using a damping and moving average.

\section{Application}

The practical applicability of the proposed scheme is investigated using the omnidirectional robot, which is kinematically able to move in any planar direction regardless of its current pose. Fig. 12 presents a photograph of the omnidirectional robot placed on the smart floor panel. During the test, the robot moved in an arbitrary trajectory over the panel surface entire course of the test.

Another possible application is to use the panel to determine the center of mass of objects. This could have practical applications in human posture analysis, such as standing posture, walking posture, and gait pattern, which is associated with alignment and balance of the human body and can be analyzed by measuring alterations of the center of gravity. Thereby, to achieve this approach to evaluate human gait, the floor panel presented in this study is extended to serve as a tool to measure the weight and center of gravity of solid objects with an irregular shape. In this case, a water bottle with symmetrical shape is utilized and favorable to analyze its center of gravity. Fig. 13 presents a 
photograph of the water bottle placed on the floor panel at the designed location with a constant coordinate position ( $\mathrm{x}=$ $y=50 \mathrm{~cm}$ ) regarding its center of gravity. The results, shown in Fig. 14, indicate that the proposed localization scheme enables the center of gravity of the water bottle to be measured and the localization resolution is approximate $\pm 1 \mathrm{~cm}$.

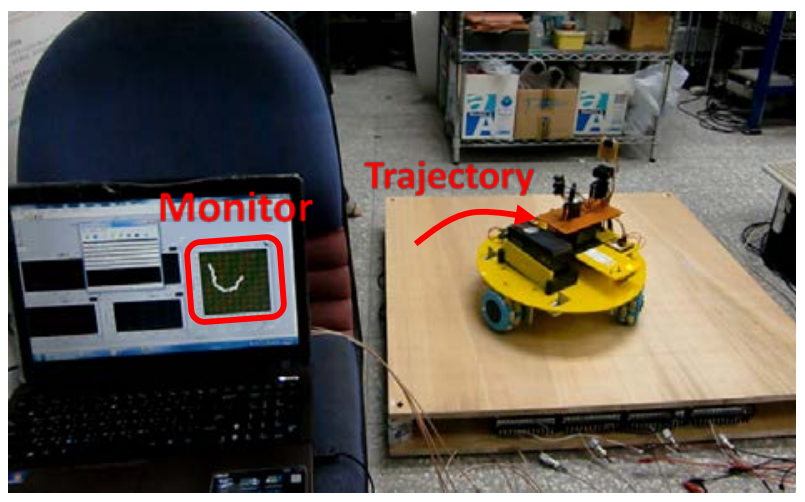

Fig. 12. The experimental setup for the practical applicability of the proposed system using an omnidirectional robot.

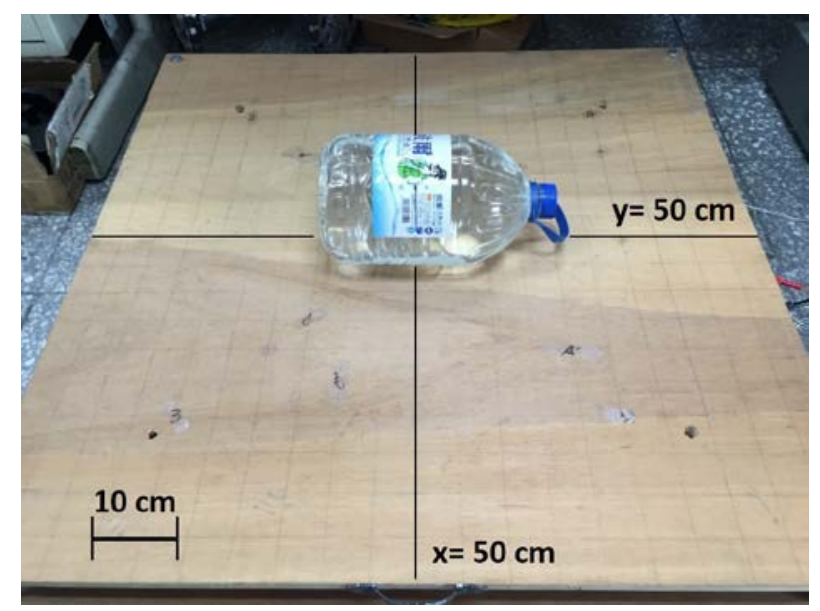

Fig. 13. The experimental setup for measuring the weight and center of gravity of solid objects with an irregular shape.

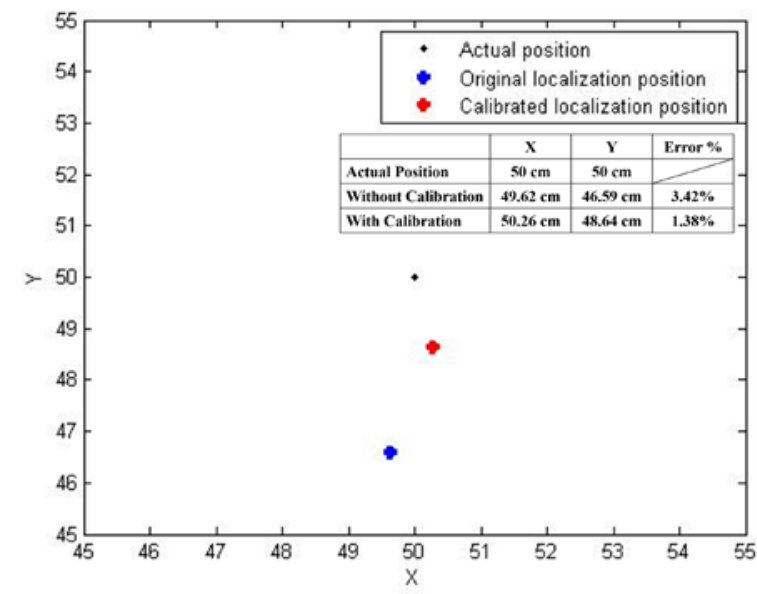

Fig. 14. The experimental results of actual position, original localization position, and calibrated localization position in center of gravity measurement tests.

\section{Discussion}

The results of the single-load static experiment have confirmed the effectiveness of the calibration system in improving the localization performance of the proposed smart load-cell based floor panel. The experimental results have shown that following the calibration process, the spatial resolution is between $0.67 \sim 2.99 \mathrm{~cm}$. Besides, the results obtained in the multi-load test have shown that the localization performance is maintained in the case where objects are placed sequentially on the panel surface and the location of each newly-placed object is determined before the next object is added. In addition, the results of a moving load experiment have also confirmed the ability of the proposed scheme to detect the motion of an object continuously. Finally, the results of the dynamic tests have shown the ability of the proposed system to achieve quasistatic states within 0.5 seconds and effectively detect the position of an individual.

In the future, based on the scenario mentioned previously and the lessons learned in this work, multiple floor panels would be integrated to form a smart floor for indoor localization. However, for real service, the system reliability and cost need to be further improved to meet the requirements. However, the study shows the proposed scheme should be a promising technology in future smart living related applications. 


\section{Conclusions}

A force-sensing floor panel has been designed and developed for the purpose of indoor localization. The weight and position of an object placed on the panel are determined based on the output of load cells mounted beneath the corners of the panel based on rigid body mechanics. A calibration algorithm has also been proposed for improving the localization accuracy of the panel. The testing results obtained from a series of single touch tests have confirmed the localization performance of the panel. The experimental results have also shown the ability of the proposed system to track the position of an object as it moves continuously on the panel surface and has been demonstrated by using an omnidirectional mobile robot placed on the panel. In addition, the floor panel can also serve as a tool to measure the center of gravity of an object for other applications. In summary, the study shows the proposed scheme should be a promising technology in future smart living related applications.

\section{Acknowledgment}

This work is supported by the ministry of science and technology (MOST) of Taiwan under contract number MOST 104-2221-E-006-121 and NSC 101-2221-E-006032-MY3.

\section{References}

(1) H. M. Khoury, and V. R. Kamat, "Evaluation of position tracking technologies for user localization in indoor construction environments," Automation in Construction, vol.18, pp.444-457, August 2009.

(2) W. Qiuping, and H. Fengtian, "New optimal approach to space-stable inertial navigation system," $201110^{\text {th }}$ IEEE International Conference on Electronic Measurement \& Instruments (ICEMI), Vol. 2, pp. 296299, 2011.

(3) N. B. Priyantha, A. K. Miu, H. Balakrishnan, and S. Teller, "The cricket compass for context-aware mobile applications," Proceedings of the $7^{\text {th }}$ annual international conference on Mobile computing and networking. ACM, pp. 1-14, July 2001.

(4) F. Gao, X. Ma, J. F. Gu, and Y. Li, "An active target localization with monocular vision," $201411^{\text {th }}$ IEEE International Conference on Control \& Automation (ICCA), pp. 1381-1386, June 2014
(5) X. Chen, L. Xie, C. Wang, and S. Lu, "Adaptive Accurate Indoor-Localization Using Passive RFID," 2013 IEEE International Conference on Parallel and Distributed Systems (ICPADS), pp. 249-256, December 2013

(6) A. Schmidt, M. Strohbach, K. Van Laerhoven, A. Friday, and H. W. Gellersen, "Context acquisition based on load sensing," UbiComp 2002: Ubiquitous Computing. Springer, pp. 333-350, 2002

(7) S. M. Killough and F. G. Pin, "Design of an omnidirectional and holonomic wheeled platform prototype," Proceedings 1992 IEEE International Conference on Robotics and Automation, pp. 84-90, May 1992

(8) W. K. Loh, K. H. Low, and Y. P. Leow, "Mechatronics design and kinematic modelling of a singularityless omnidirectional wheeled mobile robot," Proceedings 2003 IEEE International Conference on Robotics and Automation (ICRA'03), Vol. 3, pp. 3237-3242, 2003

(9) C. H. Pi, K. S. Ou, M.-H. and Chen, K.-S. Chen, "Analysis, simulation, and experimental investigations of a one dimensional touch panel based on strain sensing, " Proceedings of 50 ${ }^{\text {th }}$ SICE Annual conference on the Society of Instrument and Control Engineers of Japan, Tokyo, Japan, Sep. 2011.

(10) C. H. Pi and K. S. Chen, "A strain-sensing based scheme for indoor localization: Analysis, algorithm, and demonstration," Measurement, Vol. 51, pp. 224-235, May 2014 